\title{
Review of: "Continual reelin signaling by the prime neurogenic niche of the adult brain"
}

\author{
Inmaculada cuchillo Ibañez ${ }^{1}$
}

1 Universidad Miguel Hernández de Elche

Potential competing interests: The author(s) declared that no potential competing interests exist.

The authors address in this manuscript a topic of great interest, the role of reelin in the neurogenesis of the adult brain. This article provides new interesting data in a field that is providing very interesting articles lately. The authors use a wide methodology, such as different transgenic mice and where it is worth highlighting the use of the AP-RR36 fusion protein.

While the second part of the manuscript is better structured, the first part (Fig 1-3, Supp fig 1-3) could be improved. My main concerns is about ApoER2. Authors say that "AP-reelin staining reveals the presence of functional reelin receptors" based in their previous reports (Uchida et al., 2009; Pérez-Martínez et al., 2012). However, I think that to state that AP-RR36 leads to a colocalization with functional ApoER2 it is crucial to co-immunostay the receptor, with an antibody against LRP8/ApoER2, together with the AP-RR36 staining, if possible. Whether this is not possible, adjacent slides can be used to perform a classical IHQ using anti-reelin and anti-Apoer8 antibodies, just to check that both proteins colocalize at least.

The reasons why there are higher levels of AP-reelin staining in the Reln+/- and Reln-/- respect to those in Reln+/+ (Pag 14 and suppl 2) are not well explained.

Fig S2 should be better illustrated, indicating with arrows the regions where reelin staining appears, mainly at the Reln+/+. VLDLR-/- staining is not clear neither. Bigger photos or more contrasted would help to visualize AP-RR36 staining.

Fig 1 and Fig Supp3. Authors could consider to include an illustration or photos with lower magnification to visualize the brain areas where the different stainings belong to.

Fig 3: The addition of lines showing the total (input) and unbounds would improve the figure.

There is a mistake in References "Perez Martínez et al". It says it was published in 2012 but also "In Press".

The authors may consider to edit the English. 
